\title{
Redox imaging and optical coherence tomography of the respiratory ciliated epithelium
}

\author{
Daniel A. Gil \\ Joe T. Sharick \\ Sophie Mancha \\ Ute A. Gamm \\ Michael A. Choma \\ Melissa C. Skala
}




\section{Redox imaging and optical coherence tomography of the respiratory ciliated epithelium}

\author{
Daniel A. Gil, ${ }^{a, b}$ Joe T. Sharick, ${ }^{b, c}$ Sophie Mancha, ${ }^{a, b}$ \\ Ute A. Gamm, ${ }^{d}$ Michael A. Choma, ${ }^{\text {d,e,f,g }}$ and \\ Melissa C. Skala ${ }^{\mathrm{a}, \mathrm{b}, *}$ \\ aUniversity of Wisconsin-Madison, Department of Biomedical \\ Engineering, Madison, Wisconsin, United States \\ ${ }^{b}$ Morgridge Institute for Research, Madison, Wisconsin, United States \\ 'Vanderbilt University, Department of Biomedical Engineering, \\ Nashville, Tennessee, United States \\ 'Yale University, Department of Diagnostic Radiology, New Haven, \\ Connecticut, United States \\ eYale University, Department of Biomedical Engineering, New Haven, \\ Connecticut, United States \\ 'Yale University, Department of Pediatrics, New Haven, Connecticut, \\ United States \\ gYale University, Department of Applied Physics, New Haven, \\ Connecticut, United States
}

\begin{abstract}
Optical coherence tomography (OCT) is an emerging technology for in vivo airway and lung imaging. However, OCT lacks sensitivity to the metabolic changes caused by inflammation, which drives chronic respiratory diseases such as asthma and chronic obstructive pulmonary disorder. Redox imaging $(\mathrm{Rl})$ is a label-free technique that uses the autofluorescence of the metabolic coenzymes $\mathrm{NAD}(\mathrm{P}) \mathrm{H}$ and flavin adenine dinucleotide (FAD) to probe cellular metabolism and could provide complimentary information to OCT for airway and lung imaging. We demonstrate OCT and RI of respiratory ciliated epithelial function in ex vivo mouse tracheae. We applied $\mathrm{RI}$ to measure cellular metabolism via the redox ratio [intensity of $\mathrm{NAD}(\mathrm{P}) \mathrm{H}$ divided by FAD] and particle tracking velocimetry OCT to quantify cilia-driven fluid flow. To model mitochondrial dysfunction, a key aspect of the inflammatory process, cyanide was used to inhibit oxidative metabolism and reduce ciliary motility. Cyanide exposure over 20 min significantly increased the redox ratio and reversed cilia-driven fluid flow. We propose that $\mathrm{RI}$ provides complementary information to OCT to assess inflammation in the airway and lungs. $\odot$ The Authors. Published by SPIE under a Creative Commons Attribution 4.0 Unported License. Distribution or reproduction of this work in whole or in part requires full attribution of the original publication, including its DOI. [DOI: 10.1117/1.JBO.24.1.010501]
\end{abstract}

Keywords: redox imaging; autofluorescence; cellular metabolism; optical coherence tomography; airway; cilia.

Paper 180615LR received Nov. 7, 2018; accepted for publication Jan. 16, 2019; published online Jan. 30, 2019.

Chronic inflammation disrupts normal airway function and progressively remodels the airway, leading to the debilitating symptoms of asthma and chronic obstructive pulmonary disease (COPD). ${ }^{1}$ Our limited understanding of inflammation in these

\footnotetext{
*Address all correspondence to Melissa C. Skala, E-mail: mcskala @ wisc.edu
}

diseases has hampered drug development and left asthma and COPD patients with few effective treatments. ${ }^{2}$ Improved structural and functional imaging technologies that more comprehensively assess inflammation in disease models and patients could provide new insights into disease mechanisms and lead to new therapies.

Optical coherence tomography (OCT) provides micronscale, depth-resolved images of tissue structure and has been demonstrated for in vivo human airway and lung imaging. ${ }^{3}$ Functional extensions of OCT enable quantitative imaging of airway function (e.g., ciliary motility), structure (i.e., collagen and airway smooth muscle), and tissue remodeling associated with chronic inflammation. ${ }^{3,4}$ However, OCT lacks a contrast mechanism sensitive to the early biochemical alterations of inflammation, before immune cell recruitment or remodeling occurs.

Inflammation impairs oxidative cellular metabolism in airway cells through reactive oxygen species-induced mitochondrial dysfunction. ${ }^{5}$ Therapies that target this mitochondrial dysfunction may reduce the deleterious effects of inflammation that drive asthma and COPD progression. ${ }^{2,5}$ Functional imaging of cellular metabolism therefore would provide complementary information to OCT.

Redox imaging (RI) is a label-free technique that measures the autofluorescence of endogenous, metabolic coenzymes nicotinamide dinucleotide (NADH), nicotinamide dinucleotide phosphate (NADPH), and flavin adenine dinucleotide (FAD). NADH and NADPH have identical fluorescent properties, and, thus, are jointly referred to as $\mathrm{NAD}(\mathrm{P}) \mathrm{H}$. The redox ratio, defined as the $\mathrm{NAD}(\mathrm{P}) \mathrm{H}$ intensity divided by FAD intensity, is sensitive to the oxidation-reduction state of the cell. ${ }^{6} \mathrm{RI}$ is label-free and compatible with current bronchoscopes or modified bronchial OCT endoscopes. ${ }^{7,8}$

In this letter, we apply RI for the first time in trachea and establish feasibility of RI and OCT to assess airway function. RI and OCT were used to evaluate the effect of cyanide, an inhibitor of oxidative metabolism, on the cellular metabolism and ciliary motility of the respiratory ciliated epithelium.

All animal work was approved by the Vanderbilt University Institution Animal Care and Use Committee. Wild-type mice (female, 6 to 8 weeks, FVB/NJ, The Jackson Laboratory) were euthanized by $\mathrm{CO}_{2}$ asphyxiation followed by cervical dislocation. The trachea was excised, placed immediately into a 35$\mathrm{mm}$ dish filled with room temperature $\left(25^{\circ} \mathrm{C}\right)$ DMEM/F12 media (HEPES-buffered, no phenol red, FisherScientific), and cut along the trachealis muscle to expose the ciliated epithelium. Additional medium was used to rinse the trachea. Lastly, the trachea was transferred into a $35-\mathrm{mm}$ dish lined with cured polydimethylsiloxane (Quantum Silicones), pinned with the ciliated epithelium exposed, and covered with $1 \mathrm{~mL}$ warmed $\left(37^{\circ} \mathrm{C}\right)$ medium. The temperature was maintained with a 35 $\mathrm{mm}$ dish heater (Warner Instruments) for OCT or a stage-top incubator (Tokai Hit Co.) for RI. Ciliary motility is present after this isolation procedure.

After isolation, tracheae were assigned to one of the following treatments: (1) control: DMEM/F12 medium $(n=3$ for OCT imaging); (2) cyanide: DMEM/F12 medium supplemented with $10 \mathrm{mM}$ sodium cyanide $(n=5$ for OCT imaging and $n=5$ for RI); or (3) ethanol: a solution of $40 \%$ ethanol (200 proof, $99 \%$ pure, Sigma) and 60\% DMEM/F12 medium to de-epithelialize the tracheae ( $n=3$ for OCT imaging). 
All OCT measurements were performed with a Telesto II system (Thorlabs) with a calculated axial resolution of $4.3 \mu \mathrm{m}$ in water and a reported lateral resolution of $13 \mu \mathrm{m}$ (Thorlabs OCT-LSM03, NA 0.06). Standard OCT processing was used. For particle tracking velocimetry OCT (PTV-OCT), a 10\% suspension of $5 \mu \mathrm{m}$ polystyrene microspheres (PS05N, Bangs Laboratories) was diluted with DMEM/F12 medium for a final volume percent of $0.2 \%$ microspheres/medium, and $2 \mu \mathrm{L}$ of the final microsphere suspension was added to the $1 \mathrm{~mL}$ of medium already covering the trachea to yield $\sim 50$ microspheres per B-scan. Each B-scan comprised 800 A-lines over $4.00 \mathrm{~mm}$ (5 $\mu \mathrm{m} /$ pixel), and each PTV-OCT dataset comprised 600 repeated B-scans at the same position over $21 \mathrm{~s}$ (B-scan rate: $28 \mathrm{~Hz}$ ). To ensure no microspheres settled on the epithelial surface and interfered with ciliary beating, the following procedure was followed: (1) add microsphere suspension to the medium; (2) PTV at baseline; (3) replace medium with control, cyanide, or ethanol medium; (4) wait for $20 \mathrm{~min}$, add microsphere suspension, and perform PTV.

Streak images are the maximum intensity projection over the first $7 \mathrm{~s}$ (200 frames at $28 \mathrm{~Hz}$ ) of the PTV-OCT dataset. PTV was performed using TrackMate, a validated ImageJ/FIJI plugin for particle tracking. ${ }^{9}$ The key operating principles of TrackMate are to detect the particle, link particles across frames, and form tracks from particle links. Particle detection was achieved using the difference of Gaussians particle detector (key parameters: $5 \mu \mathrm{m}$ particle size with subpixel localization). Particle linkage and track formation were achieved using the linear motion tracker, which is based on Kalman filtering (key parameters: $5 \mu \mathrm{m}$ search radius with no frame gaps allowed). After automatically segmenting the area dominated by cilia-driven fluid flow (150 $\mu \mathrm{m}$ above the tracheal surface), each particle in this area was detected across 300 frames (B-scan rate: $14 \mathrm{~Hz}$ downsampled from $28 \mathrm{~Hz}$ ). Automated selection of particles traveling in-plane along the surface of the trachea was achieved by setting key parameters in TrackMate to select for tracks with a total displacement $>13 \mu \mathrm{m}$ (OCT lateral resolution) and duration in the field of view (FOV) $>0.35 \mathrm{~s}(5$ frames at $14 \mathrm{~Hz}$ ). Vector decomposition was used to isolate the $X$ velocity of each link's speed/angle pair in MATLAB with positive $X$ defined as the direction of overall cilia-driven fluid flow at baseline (Fig. 1). We selected the regions closest to the trachea surface and tangential flow (i.e., $X$ velocity) to isolate cilia-driven flow. PTV-OCT using TrackMate was validated using a capillary flow phantom (data not shown) and against manual particle tracking of cilia-driven fluid flow ( $n=3$, data not shown). $X$ and $Z$ velocities measured using PTV-OCT in a capillary flow phantom agreed with calculated velocities (linear regression, $r>0.99, p<0.001$ ). Agreement was also achieved between automated tracking using TrackMate and manual tracking in untreated trachea $(n=3$, $p>0.05)$.

All RI measurements were performed using an upright epifluorescence microscope (Nikon Ni-U). Systems specifications include a $4 \times$ air objective (Nikon CFI Plan Fluor, NA 0.13, FOV: $1.65 \mathrm{~mm} \times 2.2 \mathrm{~mm}$, lateral resolution: $2.16 \mu \mathrm{m}$ at $460 \mathrm{~nm} / 2.46 \mu \mathrm{m}$ at $525 \mathrm{~nm}$ ), a white-light LED source (XCite 120LED), and a cooled $\left(-20^{\circ} \mathrm{C}\right.$ ) interline CCD (Andor Clara E, image pixel size: $1.58 \mu \mathrm{m})$. NAD(P)H fluorescence was excited with a DAPI filter cube (Nikon, ex: 361 to $389 \mathrm{~nm} / \mathrm{em}$ : 435 to $485 \mathrm{~nm}$ ) and integrated over $2 \mathrm{~s}$ (power at $375 \mathrm{~nm}$ : $1.6 \mathrm{~mW}$ ). FAD fluorescence was excited with a

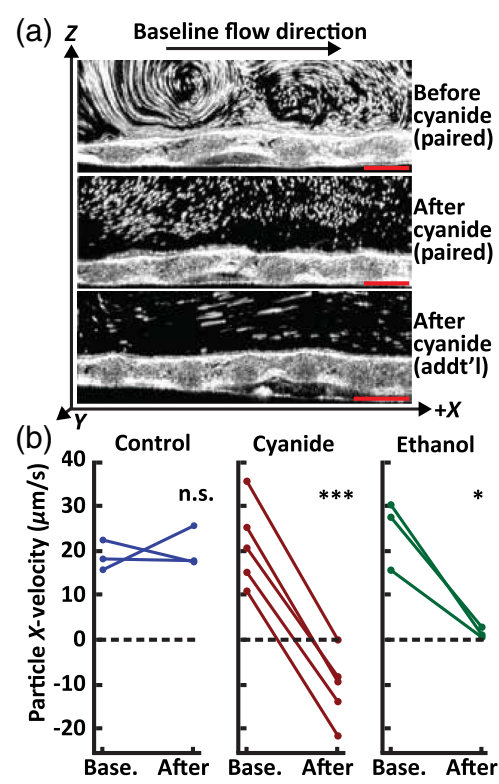

Fig. 1 PTV-OCT. (a) Streak images show reduced cilia-driven fluid flow 21 min postcyanide treatment compared to baseline in $n=1$ paired sample. An additional streak image is shown to capture the variability in particle $X$ velocity postcyanide treatment. Direction of baseline cilia-driven fluid flow is defined as $+X$. Scale bar: $500 \mu \mathrm{m}$. (b) Particle $X$ velocity at baseline and after no treatment (control, n.s., $n=3$ ), cyanide treatment ( ${ }^{* *}$ for $\left.p<0.001, n=5\right)$, and de-epithelialization with ethanol ( ${ }^{*}$ for $p<0.05, n=3$ ).

GFP filter cube (Nikon, ex: 450 to $490 \mathrm{~nm} / \mathrm{em}: 500$ to $550 \mathrm{~nm}$ ) and integrated over $6 \mathrm{~s}$ (power at $450 \mathrm{~nm}: 1.27 \mathrm{~mW}$ ). The following protocol was followed: (1) RI at baseline, (2) replace medium with cyanide medium, and (3) RI 1 min after treatment and then every $2 \mathrm{~min}$ for $20 \mathrm{~min}$. Redox ratio images were calculated per-pixel by dividing the $\mathrm{NAD}(\mathrm{P}) \mathrm{H}$ image by its corresponding FAD image (ImageJ). Two regions-of-interest (ROI: cartilage and noncartilage) were manually segmented using the $\mathrm{NAD}(\mathrm{P}) \mathrm{H}$ intensity image, in which the tracheal hyaline cartilage is visible. ${ }^{10}$ This mask was then applied to the corresponding $\mathrm{NAD}(\mathrm{P}) \mathrm{H}, \mathrm{FAD}$, and redox ratio image time series (ImageJ). The mean value was calculated for each ROI per image. A control experiment (no cyanide) confirmed the RI protocol caused no photobleaching (data not shown).

$\mathrm{RI}$ and PTV-OCT measurements were performed on separate samples on separate imaging systems, so no image coregistration was used. Linear mixed-effect (LME) models were used to analyze RI and PTV-OCT data and assess cyanide treatment response (nlme, R). LME models allow for differences in baseline measurements due to inherent sample-to-sample variation. For RI time-series data, a first-order autoregressive [AR(1)] covariance structure was used and pairwise comparisons between the two regions (cartilage and noncartilage) were also performed at every time point using least-squares means (lsmeans, R). Normalized values were calculated as $X_{\text {post-treatment }} / X_{\text {baseline, }}$, where $X$ is redox ratio, NAD $(\mathrm{P}) \mathrm{H}$ intensity, and FAD intensity.

Representative streak images (integrated time series) shown in Fig. 1(a) qualitatively demonstrate the reduction in ciliadriven fluid flow due to cyanide treatment. Quantitative changes in cilia-driven particle velocity across treatment conditions are shown in Fig. 1(b). Control treatment over 20 min caused no change in $X$ velocity. Cyanide treatment over 20 min caused 

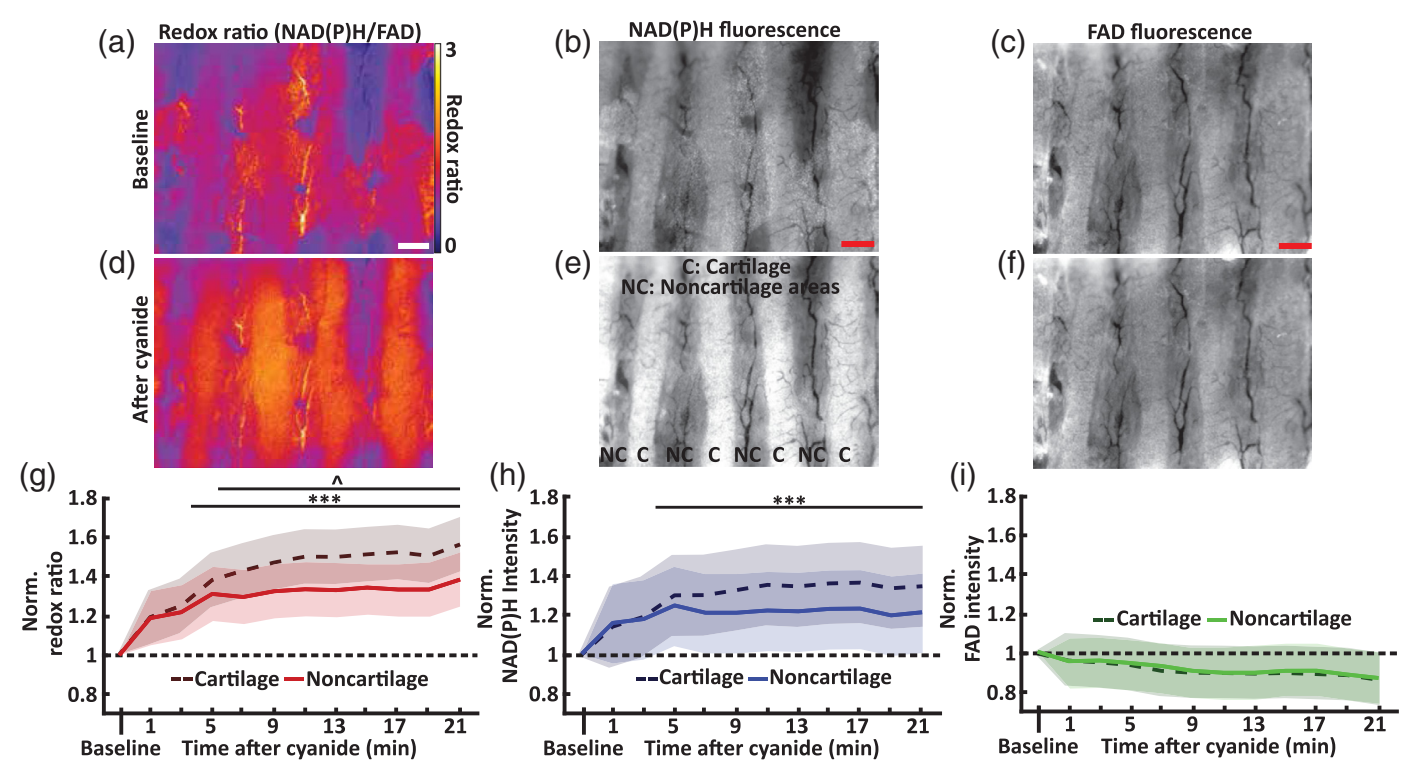

Fig. 2 Redox imaging. Redox ratio images (a) before and (d) 21 min after cyanide treatment. NAD(P)H images (b) before and (e) 21 min after cyanide treatment. Regions with cartilage, "C," and noncartilage, "NC," regions can be discriminated. FAD images (c) before and (f) 21 min after cyanide treatment. Scale bar: $500 \mu \mathrm{m}$. (G) By $5 \mathrm{~min}$, the redox ratio significantly increased for both the cartilage and noncartilage regions compared to baseline $\left({ }^{* \star \star}\right.$ for $\left.p<0.001, n=5\right)$. Beginning at $7 \mathrm{~min}$, the redox ratio of the cartilage region was significantly higher than the noncartilage region ( $\wedge$ for $p<0.05$ ). (h) By 5 min, NAD(P)H intensity significantly increased in both the cartilage and noncartilage regions compared to baseline $\left({ }^{\star \star \star}\right.$ for $p<0.001$ ). (i) Cyanide treatment resulted in no significant change in FAD intensity in both regions compared to baseline. Shaded region in $(\mathrm{g}),(\mathrm{h})$, and (i) is standard error of the mean.

a reversal of flow direction (i.e., negative $X$ velocities), and in 4 out of 5 samples a decrease in the $X$ velocity magnitude. Finally, de-epithelialization with ethanol treatment caused a large and significant decrease in $X$ velocity.

Representative images of the redox ratio, NAD $(\mathrm{P}) \mathrm{H}$ fluorescence, and FAD fluorescence before and 21 min after cyanide treatment are shown in Figs. 2(a)-2(f). Qualitatively, these representative images indicate a large increase in redox ratio [Figs. 2(a) and 2(d)] and NAD(P)H intensity [Figs. 2(b) and 2(e)] due to cyanide treatment. Quantitative analysis of the RI time-series data shows a significantly increased redox ratio $(p<0.001)$ and $\mathrm{NAD}(\mathrm{P}) \mathrm{H}$ intensity $(p<0.001)$ for both cartilage and noncartilage regions after $5 \mathrm{~min}$ of cyanide treatment [Figs. 2(g) and 2(h)], respectively. The cartilage regions had a larger increase in redox ratio compared to noncartilage regions starting at $7 \mathrm{~min}$ after cyanide treatment [Fig. 2(g)]. The increase in the redox ratio is due to a significant increase in $\mathrm{NAD}(\mathrm{P}) \mathrm{H}$ intensity [Fig. 2(h)] and small but nonsignificant decrease in FAD intensity [Fig. 2(i)].

Quantitative comparison of the change in particle velocity and redox ratio caused by $20 \mathrm{~min}$ of cyanide treatment is as follows. Cyanide treatment caused a decrease in particle $X$ velocity of $-219 \%$ (95\% CI: $[-309 \%-130 \%])$ and an increase in redox ratio of $47 \%$ (95\% CI: [39\% 55\%]) across both cartilage and noncartilage regions. By region, redox ratio increased $55 \%$ in cartilage regions (95\% CI: [32\% 79\%]) compared to $38 \%$ in noncartilage regions (95\% CI: [12\% 63\%]).

Here, RI and OCT were applied, for the first time, to assess the ciliated epithelium of ex vivo mouse tracheae, a well-characterized model of human respiratory biology. Large animal models are often used to study airway physiology, but we chose the mouse trachea for this initial study because mice are widely used in studies that require genetic modification and/or large numbers of samples at a reasonable cost. ${ }^{11}$ Inflammation in the airway impairs oxidative cellular metabolism through reactive oxygen species-induced mitochondrial dysfunction. To simulate mitochondrial dysfunction, we treated the tracheae with cyanide, which inactivates cytochrome $\mathrm{C}$ oxidase (complex IV) to inhibit the electron transport chain (ETC) and oxidative phosphorylation. Cyanide is commonly used as a validation for RI. ${ }^{12}$ Additionally, Pseudomonas aeruginosa, a bacterium that causes ventilator-associated pneumonia and can be found in the lungs of cystic fibrosis patients, excretes cyanide in the form of hydrogen cyanide. ${ }^{13}$

Oxidative phosphorylation is the most efficient metabolic pathway to generate ATP. In ciliated epithelial cells, ATP powers the coordinated action of multiple dynein motor proteins that drive ciliary beating. Thus, inhibiting oxidative metabolism in ciliated cells affects ciliary motility. We applied PTV-OCT to measure cilia-driven fluid flow (i.e., the output of ciliary motility) before and after cyanide treatment. Cyanide led to a reversal of the flow direction [Figs. 1(a) and 1(b)], which indicates ciliadriven fluid flow that is ineffective for mucus clearance. Previous studies have shown that cyanide can alter levels of intracellular calcium and that altered calcium levels cause a reversal in the ciliary beat pattern in Paramecium, ${ }^{14,15}$ which is consistent with our observation of reversed flow direction with cyanide treatment. De-epithelialization with ethanol resulted in a cessation of cilia-driven fluid flow [Fig. 1(c)] with no flow reversal.

In parallel, we applied RI to assess the metabolic response to cyanide treatment. The ETC uses NADH and FAD as electron donors and acceptors, respectively. Thus, inhibition of the ETC with cyanide reduces NADH consumption and FAD generation. The significant increase in the redox ratio [Figs. 2(a) and 2(d)] is primarily driven by increased $\mathrm{NAD}(\mathrm{P}) \mathrm{H}$ intensity [Figs. 2(b) 
and 2(e)] and a small decrease in FAD intensity [Figs. 2(c) and 2(f)], as previously reported. ${ }^{12}$ After correcting for differences in baseline fluorescence, regions above the cartilage had a larger increase in redox ratio with cyanide treatment compared to noncartilage regions [Fig. 2(g)]. The wavelengths used for RI integrate over $\sim 200$ to $300 \mu \mathrm{m}$, heavily weighted for the superficial layers, and the thickness of mouse tracheae is 200 to $250 \mu \mathrm{m} .{ }^{16}$ Therefore, we attribute this increased change in redox ratio of cartilage regions to the additional metabolically active cells (chondrocytes) present below the epithelia of the cartilage regions that are not present in noncartilage regions. ${ }^{10}$

Finally, we compared the change in cellular metabolism and cilia-driven fluid flow caused by cyanide treatment. These results indicate that, when oxidative phosphorylation is inhibited in ciliated epithelial cells with cyanide (increased redox ratio), there is reduced capacity to generate effective (i.e., $+X$ velocity) cilia-driven fluid flow. This suggests that $\mathrm{RI}$ is sensitive to the metabolic stimuli linked to ciliary motility as well as the overall metabolic state of cells within the airway.

We believe these results merit future development of combined RI and OCT of the airway as they provide complementary functional information. Preclinically, RI and OCT could be combined to offer insights into airway disease pathogenesis. In the clinic, existing bronchoscopes or multimodal OCT endoscopes could incorporate RI to monitor early inflammation in patients and assess the efficacy of therapies. Pahlevaninezhad et al. ${ }^{8}$ have previously demonstrated in vivo autofluorescence imaging and OCT using a minimally invasive probe, and a similar design could be used to perform our measurements in vivo in patients. Additional improvements for combined OCT and RI include incorporating a label-free OCT method to assess ciliary motility (e.g., speckle monitoring of ciliary beat frequency) and performing RI with optical sectioning (e.g., confocal, multiphoton, or structured illumination microscopy).

\section{Disclosures}

The authors declare that there are no conflicts of interest related to this letter.

\section{Acknowledgments}

The author would like to thank J. Eickhoff for assistance with statistical analysis and M. Lapierre-Landry, T. Heaster, and A. Gillette for their useful discussions.

\section{References}

1. P. J. Barnes, "Immunology of asthma and chronic obstructive pulmonary disease," Nat. Rev. Immunol. 8(3), 183-192 (2008).

2. N. J. Gross and P. J. Barnes, "New therapies for asthma and chronic obstructive pulmonary disease," Am. J. Respir. Crit. Care Med. 195(2), 159-166 (2017).

3. S. D. Murgu et al., "Optical coherence tomography in pulmonary medicine," in Optical Coherence Tomography: Technology and Applications, pp. 2263-2303, Springer, Cham (2015).

4. B. K. Huang and M. A. Choma, "Microscale imaging of cilia-driven fluid flow," Cell Mol. Life Sci. 72(6), 1095-1113 (2015).

5. Y. S. Prakash, C. M. Pabelick, and G. C. Sieck, "Mitochondrial dysfunction in airway disease," Chest 152(3), 618-626 (2017).

6. K. P. Quinn et al., "Quantitative metabolic imaging using endogenous fluorescence to detect stem cell differentiation," Sci. Rep. 3, 3432 (2013).

7. Q. He et al., "Value of autofluorescence imaging videobronchoscopy in detecting lung cancers and precancerous lesions: a review," Respir. Care 58(12), 2150-2159 (2013).

8. H. Pahlevaninezhad et al., "Endoscopic high-resolution autofluorescence imaging and OCT of pulmonary vascular networks," Opt. Lett. 41(14), 3209-3212 (2016).

9. J. Y. Tinevez et al., "TrackMate: an open and extensible platform for single-particle tracking," Methods 115, 80-90 (2017).

10. M. Navarro, J. Ruberte, and A. Carretero, "Respiratory apparatus," in Morphological Mouse Phenotyping, J. Ruberte, A. Carretero, and M. Navarro, Eds., pp. 147-178, Academic Press, Cambridge, Massachusetts (2017).

11. G. M. Solomon et al., "Assessment of ciliary phenotype in primary ciliary dyskinesia by micro-optical coherence tomography," JCI Insight 2(5), e91702 (2017)

12. A. J. Walsh et al., "Optical metabolic imaging identifies glycolytic levels, subtypes, and early-treatment response in breast cancer," Cancer Res. 73(20), 6164-6174 (2013).

13. C. Nair et al., "Cyanide levels found in infected cystic fibrosis sputum inhibit airway ciliary function," Eur. Respir. J. 44(5), 1253-1261 (2014).

14. Y. Iwadate, "Photolysis of caged calcium in cilia induces ciliary reversal in Paramecium caudatum," J. Exp. Biol. 206(Pt. 7), 1163-1170 (2003).

15. Y. X. Wang et al., "Metabolic inhibition with cyanide induces calcium release in pulmonary artery myocytes and Xenopus oocytes," Am. J. Physiol. Cell Physiol. 284(2), C378-C388 (2003).

16. S. L. Jacques, "Optical properties of biological tissues: a review," Phys. Med. Biol. 58(11), R37-R61 (2013). 